Mots. Les langages du politique

$78 \mid 2005$

Usages politiques du genre

\title{
De Barbare à Babel, des sons qui bredouillent et excluent
}

Maurice Tournier

\section{OpenEdition}

Journals

Édition électronique

URL : https://journals.openedition.org/mots/446

DOI : $10.4000 /$ mots.446

ISSN : 1960-6001

Éditeur

ENS Éditions

Édition imprimée

Date de publication : 1 juillet 2005

Pagination : 131-142

ISBN : 2-84788-080-1

ISSN : 0243-6450

Référence électronique

Maurice Tournier, «De Barbare à Babel, des sons qui bredouillent et excluent », Mots. Les langages du politique [En ligne], 78 | 2005, mis en ligne le 31 janvier 2008, consulté le 24 avril 2022. URL : http:// journals.openedition.org/mots/446; DOI : https://doi.org/10.4000/mots.446 


\title{
Maurice Tournier
}

\section{DES MOTS EN POLITIQUE}

\section{De Barbare à Babel, des sons qui bredouillent et excluent}

\begin{abstract}
L'analyse externe et l'analyse interne ne sont nullement en contradiction, mais reflètent cependant deux attitudes, deux approches, deux angles de vue différents. La première cherche d'abord l'étymon historique et n'admet une origine onomatopéique qu'en dernier ressort, alors que nous postulons d'abord la structure onomatopéique et cherchons ensuite le mot dans lequel elle s'est actualisée.
\end{abstract}

(Pierre Guiraud) $^{1}$

Parmi les « structures onomatopéiques » des mots français, que Pierre Guiraud a si bien mises en évidence au-delà des étymons philologiques et des facteurs historiques, il en est une sur laquelle il n'est pas sans intérêt de s'interroger: la séquence BR/BL. Contradictoire dans sa structure, cette suite est formée par une occlusive bilabiale sonore nettement articulée suivie de son contraire, deux constrictives linguales dites vibrante ou latérale, "appelées liquides par les grammairiens de l'Antiquité parce que leur base d'articulation est plus souple, plus élastique et, partant, plus inconsistante que celle des autres consonnes $»^{2}$. La liaison des deux types de phonèmes, bref et continu, n'engendrerait-elle pas une résultante chaotique, mélangée et indécise, peu aisée à prononcer, à entendre, à décrypter, comme si la seconde consonne venait brouiller, ébranler, broyer, briser l'effet de la première?

Il faut, comme P. Guiraud, partir de constats. Nous prendrons pour champ d'investigation, non pas les milliers de formes du Französisches Etymologisches Wörterbuch ${ }^{3}$, mais simplement quelques exemples proposés par le Robert historique 4 . Nombreux sont les mots "isosémantiques », initiés par

1. P. Guiraud, 1967, Structures étymologiques du lexique français, Paris, Larousse, p. 85 .

2. M. Niederman, 1953, Phonétique historique du latin, Paris, Klincksieck, p. 100.

3. Wartburg (von) W., 1949, Französisches Etymologisches Wörterbuch, Tübingen, J. C. B. Mohr.

4. A. Rey éd., 1995, Dictionnaire historique de la langue française, Paris, Dictionnaires Le Robert, nouvelle édition, 2 vol. (désormais : Robert historique). 
$B R / B L$ ou d'autres combinaisons du même genre, qui évoquent comme thèmes permanents le bégaiement, l'incompréhension, l'inculte, l'étranger, le menteur, le sauvage.

On glose ces actes de communication manqués par: s'exprimer avec maladresse, parler du nez, parler entre ses dents ou dans sa barbe, faire des bruits d'eau, de boue, de bourbe, de bouillon, de mare ou clabauder dans la vase comme une oie, parler comme une chèvre, un chameau, un âne, tout mélanger, sons et couleurs, parler à tort et à travers et, à la limite, tromper... Toujours le même type de consonances proches et contraires dans tous ces cas. Quelques exemples de mots bâtis sur une suite des deux consonnes B puis R ou L, avant d'aller jusqu'aux hypothèses.

\section{Séquences à deux consonnes}

Prenez des verbes en BR (ou B-R), échangez leur finale avec le suffixe péjoratif ouille (hypothèse Guiraud) et vous obtenez toute la série, apparue au $13^{\mathrm{e}} \mathrm{S}$., des bredouiller, issu de bredeler, "marmotter rapidement» (Robert historique), brouiller ${ }^{5}$ repris de broillier, mélanger, brouillas, brouillard, brouillasse, brouillon, brouillamini ${ }^{6}$, tout en confusion. Quelles origines ? L'argile, la boue, la berdouille ou la brai, brau, broi (boue, $12^{\mathrm{e}} \mathrm{s}$.), ou encore le breu/brou (13 ${ }^{\mathrm{e}} \mathrm{s}$.), et leur diminutif brouet (bouillon), issus du germanique brod qui aurait donné aussi broe, brouée ou bérouée (bruine), peut-être brouer et embrouer7, tous lieux ou actions propices au bredouillage, à la confusion comme au pataugeage. Enfin, à propos des matières évoquées par le couple BR, il reste la plus sale : le bran ou bren $\left(12^{\mathrm{e}} \mathrm{s}\right.$.), mot-injure tant aimé de Rabelais, qui viendrait du gaulois par le latin vulgaire brenum, pour désigner soit le rebut du blé, le son, soit les déchets de l'homme, ordures et excréments. De là est issu au $15^{\mathrm{e}} \mathrm{s}$. le verbe brener (et embrener), dont par métathèse on aurait fait berner, duper (hypothèse John Orr). On n'en tire rien : bernique (brenicle, $18^{\mathrm{e}} \mathrm{s}$.).

Bref, le voyageur s'y perd, comme dans le secouement des vagues, la bourlingue, dans le brouhaha biblique comme dans le bordel moderne ou dans l'inculte broussaille (broçaille, $12^{\mathrm{e}} \mathrm{s}$.). Même avec les voyelles les plus aigües, il n'entend que bris et bruits et ne distingue que brume et bruine. Et

5. Au sens de s'agiter en tous sens, en désordre. Le chœur des Juives (1583) de Robert Garnier se lamente ainsi: "Rien d'arresté ne se voit en ce monde/On y brouille toujours.» (1945, Les Juives, Paris, Garnier frères, acte IV, p. 89).

6. Brouillamini, la confusion sonore chez Molière: «Trop de tintamarre là-dedans, trop de brouillamini » (Le Bourgeois gentilhomme, acte II, scène 4). De bouliaminy, terre argileuse, latin boli armenii, boule d'Arménie (condiment indigeste?), mot burlesque selon Trévoux (Robert historique).

7. Villon: «Brouez, benards [...] Coquille ci s'embroue!» (remuez-vous, imbéciles! [...] Les voleurs ici s'empêtrent dans le brouillard), «Ballade IV », Manuscrit de Stockholm, F. Villon, 1962, Euvres, Paris, Garnier, p. 182. 
l'étymologiste se perd avec lui. Tant d'origines différentes sont proposées pour une même séquence consonantique de valeur sémantique et affective équivalente!

Un malaise identique à l'audition, allant jusqu'au soupçon de tromperie, se retrouve dans barragouyn (BR), attesté au $14^{\mathrm{e}} \mathrm{s}$. Il s'agirait là de Bretons (un verbe bretonner aurait signifié "parler français comme un Breton ») ${ }^{8}$ : car, sur les routes des pèlerinages, ces «estrangers » entraient dans les auberges en réclamant «Bara, Gwin » (pain, vin). C'est du moins Albert Dauzat qui l'assure. Mais une autre étymologie est possible, le gouin est un lourdaud qu'on pourrait aisément tromper (barat) : le baragouin $\left(16^{\mathrm{e}} \mathrm{s}\right.$.), langage de et pour les lourdauds? Un baragouineux est défini par Richelet (Dictionnaire, 1680) comme : «Celui qui baragouine, qui parle un certain jargon qu'on n'entend pas bien.» À propos d'un gascon qui «a la mine d'un étranger», Scapin s'écrie: «Ah! Peste soit du baragoüineux! » (Molière, Fourberies de Scapin, acte III, scène 2). Baragouin rend compte aujourd'hui « d'une branche inférieure et rabougrie de l'arbre des langues du monde ${ }^{9}$.

N'oublions pas, à propos de barat, que la séquence BR se retrouve dans barater/bareter, verbe attesté chez Froissart ( $14^{\mathrm{e}} \mathrm{s}$.) au sens tromper, frauder. Un baretere est un conteur de sornettes, même s'il s'agit d'un archiprêtre:

Mes est li Arceprestes si grans bareteres que, se il venoit jusques a nous en nous comptant gengles et bourdes, [...] si nous poroit tourner a grant contraire ${ }^{10}$.

On sait que «Charabarat » est le nom populaire donné à un très ancien marché aux chevaux de Lyon, issu de deux verbes (charrer et barater) qui signifient l'un et l'autre «mener en bateau» : d'où ce marché doublement trompeur à la réputation douteuse ${ }^{11}$... N'oublions pas que, à la suite de barat, est venu baratin, mot d'origine provençale, dit-on, qui signifie tromperie au $15^{\mathrm{e}} \mathrm{s}$. Baratiner et baratineur, eux, sont tout récents, mais la logorrhée est restée la même. Embarras ( $16^{\mathrm{e}} \mathrm{s}$.), encombrement, viendrait, lui, par l'espagnol d'un verbe galicien embaraçar, "dérivé de baraçao, courroie, corde, d'origine incertaine » (Robert historique). Pour leur part, une bourde $\left(12^{\mathrm{e}} \mathrm{s}\right.$. , "origine obscure», avoue le Robert historique) a d'abord désigné un mensonge et un bourdon une «histoire inventée pour abuser de la crédulité $»^{12}$.

Les bruits et cris d'animaux sont aussi incompréhensibles par nature. Le désignant bourdon n'aurait-il pas d'abord rendu compte du «bruit fait par l'insecte

8. Un vieux verbe breter, marmonner, a peut-être servi de base à un greffon «breton », par analogie (une bretete est une écervelée, un brecton un rot, un breton un voleur).

9. L.-F. Prudent, 1980, Des baragouins à la langue antillaise, Paris, Éditions caribéennes, p. 7.

10. Récit de la bataille de Cocherel (1364), cité dans G. Paris et A. Jeanroy éd., 1948, Extraits des chroniqueurs français, Paris, Hachette, p. 230.

11. On lit dans Le Livre des mestiers d'Estienne Boileau $\left(13^{\mathrm{e}} \mathrm{s}\right.$.) : «En teus marchiés [il y ] a trop de baras. »

12. Signalons aussi le sens technique de bourdon en imprimerie: «omission d'un mot ou d'un membre de phrase» (Robert historique). 
en volant »; côté humain, bourdonner ( $17^{\mathrm{e}} \mathrm{s}$.), c'est, sans parole audible, «chanter à bouche fermée » (Robert historique). Braire, brailler, bramer, bruire, après avoir signifié crier $^{13}$, rugir, mugir, faire du bruit, ont concerné l'âne ou le cerf, voire le corbeau du Roman de Renart. Les étymologistes proposent toutes sortes de racines linguistiques en invoquant un latin populaire non attesté ( ${ }^{\star}$ bragere pour braire, *bragulare pour brailler, *brugere pour bruire) ou le gotique ( ${ }^{\star} b r a m o n$ pour bramer). Le beroard ou berard de Villon est une injure rappelant le hurlement du loup-garou, nommé bérou, et barbeu depuis le $13^{\mathrm{e}} \mathrm{s}$.

Il en est de même pour la séquence BL. Beugler (bugler, $12^{\mathrm{e}} \mathrm{s}$. $)^{14}$ trouve son origine dans bugle, jeune bœuf (latin buculus). Le latin balare, qui signifiait bêler et dire des sottises, a fourni notre français bêler $\left(12^{\mathrm{e}} \mathrm{s}\right.$.), cri de la chèvre, de la brebis (du vieux latin berbex, mouton, surnommé balans, celui qui bêle, par Virgile) et du bélier, ancien belin, surnom du mouton dans le Roman de Renart; le verbe blaterare a rappelé en latin le chameau, voire la grenouille (appelée barbelote, la petite barbue, chez Chrestien de Troyes), avant de signifier bavarder et, lui aussi, dire des sottises : d'où, par emprunt au $17^{\mathrm{e}} \mathrm{s}$., blatérer, puis déblatérer ( $18^{\mathrm{e}} \mathrm{s}$.), parler à tort et à travers (blatire, chez Plaute, c'était dire des riens).

On trouve aussi, avec BL en initiale, au $13^{\mathrm{e}} \mathrm{s}$., l'adjectif et substantif bles, blois, pour bègue, et les verbes bléser, bloiseer $\left(12^{\mathrm{e}} \mathrm{s}\right.$.), pour « parler avec un défaut d'articulation » (Robert historique) - du latin blaesus, qui balbutie -, ainsi que dans la famille d'ancien français bole, boule, bulle, bouler, renverser, bolir, boulir, faire des bulles $\left(11^{\mathrm{e}} \mathrm{s}\right.$.), à rattacher au latin bulla, «d'origine expressive sinon onomatopéique » (ibid.), bullire, qui a donné boulu, boulon, boillon, bouillon, bouillis au $12^{\mathrm{e}} \mathrm{s}$. Additionné à son compère tautologique verser, le verbe bouler donne bouleverser ( $16^{\mathrm{e}} \mathrm{s}$.), mettre sens dessus dessous. Enfin, n'oublions pas que BL, origine de bulle comme de balle et autres produits du gonflage ou de l'enflure, aurait donné blague (blaque, $18^{\mathrm{e}} \mathrm{s}$.), blaguer et blagueur ${ }^{15}$ Le mensonge par la confusion...

\section{Séquences à trois consonnes}

Dans les termes déjà évoqués, nous avons vu passer quelques suites plus complexes, telles que baretere, barbeu, barbelote, bouleverser, blatérer, ébranler. Examinons d'abord la séquence BRB. Barboter initialement (12 ${ }^{\mathrm{e}} \mathrm{s}$.)

13. «Quand je vois tout nus ces truands [...] De froid, de faim crier et braire... » (Roman de la Rose, $\left.13^{\mathrm{e}} \mathrm{s}.\right)$; «Tiercelin [...] jette un bret» (Roman de Renart, $12^{\mathrm{e}} \mathrm{s}$.).

14. Qui aboutira au beuglant, café-concert $\left(19^{\mathrm{e}} \mathrm{s}\right.$.).

15. «Rengainez votre phraséologie! [...] Avec tout ce tintamarre de démagogues, vous n'avez été jusqu'à présent que des blagueurs » (Proudhon, Le Peuple, 1850); «Les nationalités! Ceci est de tradition, c'est-à-dire de blagologie révolutionnaire » (Proudhon, Lettre à Charles-Edmond, 1854). 
voulait dire "parler entre ses dents» (Robert historique). Ce pourrait être aussi parler dans sa barbe, si l'on accepte l'hypothèse d'un «croisement » du radical dit onomatopéique avec le mot barbe, hérité du latin barba (Robert historique). Quant au sens de cacher ou de chercher ce qui est dissimulé, il est plus tardif: c'est ainsi qu'un barbot est, en argot, devenu un vol et la barbote, la fouille d'un détenu (Vidocq, 1835). BRB embrouille les gens dans des secrets, ceux des barbouzes, par exemple, cachés derrière leur fausse barbe.

Pour certains étymologistes, il s'agit là aussi d'eau plus ou moins propre, endroit clapotant où l'on barbote, patauge, en agitant mains ou pieds ( $16^{\mathrm{e}} \mathrm{s}$.). La parole difficile y prend image. La borbe $\left(13^{\mathrm{e}} \mathrm{s}\right.$ ) devenue bourbe $\left(14^{\mathrm{e}} \mathrm{s}\right.$.), fange épaisse (héritée d'un gaulois *borvo, selon le Robert historique), le borbier $\left(13^{\mathrm{e}} \mathrm{s}\right.$.) ou bourbier $\left(14^{\mathrm{e}} \mathrm{s}\right.$.), une barbote (mare), un barbot (creux fangeux), etc. Cela produit, avec l'inévitable suffixe -ouille, le verbe barbouiller $\left(15^{\mathrm{e}} \mathrm{s}\right.$.), au sens originel de bredouiller, bafouiller. Le sème salir, souiller, ne devient majeur dans ces verbes que plus tard, et encore le Dictionnaire de l'Académie de 1884 confère-t-il à barbouilleur, parmi d'autres valeurs, celle de bavard (cit. : «Faites taire ce barbouilleur!»). De borbe/bourbe (d'où l'on a pu dégager une racine indo-européenne *bher- au sens de bouillonner) viennent borboter/bourbouter ( $13^{\mathrm{e}} \mathrm{s}$.), où le barbouteur ( $16^{\mathrm{e}} \mathrm{s}$.) a d'abord pataugé dans ses mots. Reste la vase. Les oies y font du bruit quand elles la fouillent de leur bec: d'où le mot-valise qui unit, dans le parler lyonnais du $18^{\mathrm{e}} \mathrm{s}$., barbouiller et fouiller (ou, mieux, farfouiller) sous la forme de barfouiller, qui donnera le français bafouiller, au sens de "parler d'une manière confuse, incohérente et peu intelligible» (19 $\mathrm{e}$ s., Robert historique). En ancien lyonnais, un barjafle parle à tort et à travers: les sons lui sortent de partout, selon de multiples types de constriction $(r, j, f, l)$ qui noient l'occlusion initiale.

La séquence BLB est, elle, à l'origine du latin balbus, qui signifie bègue (Horace parle des balba verba, paroles balbutiées ; Cicéron utilise le verbe balbutire pour parler sans suite) ${ }^{16}$. De balbus viennent directement les termes du vieux français bauber et baube (attestés au $13^{\mathrm{e}} \mathrm{s}$. au sens de bégayer et de bègue), tandis que descendent, par l'entremise d'un latin populaire supposé *balbutiare, nos balbutier ( $14^{\mathrm{e}} \mathrm{s}$.), balbutie ( $16^{\mathrm{e}} \mathrm{s}$.) puis balbutiement $\left(18^{\mathrm{e}} \mathrm{s}\right.$.).

Le mélange désordonné ne s'est pas fait que dans les bruits et les mots. II s'étend à l'écriture, à la peinture, à la vue. Séquence BRB : barbouiller, qui ne signifiait que mal parler, s'emploiera aussi pour mal écrire au $16^{\mathrm{e}}$ et pour mal peindre au $17^{\mathrm{e}}$. Un barbouteur devenu barbouilleur pataugera dans les couleurs comme dans les graphies. Séquence BRL: branler (bransler, $11^{\mathrm{e}} \mathrm{s}$. , secouer), c'est par la suite bouger en se dandinant, (s')agiter en tous sens et, au figuré, n'être pas sûr de ce qu'on dit ou pense. Mêmes valeurs dans le

16. Rapprochements dans le Robert historique: le russe balabolka désigne un «moulin à paroles » (rapproché à son tour de balalaïka). 
boulonnais barloquer, berloquer, osciller jusqu'à s'endormir ${ }^{17}$. On retrouve cette séquence dans baricoula, provençal, pour signifier «parler inconsidérément», ce verbe étant à relier, dit le Robert, au français barriolé, où les couleurs comme les sons se mélangent. BRL fait aussi la base de berlue (16 $\mathrm{s}$.), de l'ancien verbe berluer ou éberluer (éblouir), qui rend compte d'"une impression visuelle trompeuse " (Robert historique). Dans le Jargon de l'argot réformé (1836), berlu va jusqu'à signifier aveugle ${ }^{18}$.

Il existe aussi les suites BBR et BBL. Qu'est-ce qu'un bobard (1900), sinon, au départ, celui qui raconte une fausse nouvelle (un bobert au $13^{\mathrm{e}} \mathrm{s}$. était le vaniteux qui faisait la moue, $b o b$ ) ? Quant à BBL, cette séquence est quasi originelle puisque l'enfant la pratique en premier: il babille $\left(12^{\mathrm{e}} \mathrm{s}\right.$.); on est suspendu à son babil $\left(15^{\mathrm{e}} \mathrm{s}\right.$.) sans arriver à le comprendre; cela dans nombre de langues: le Robert cite l'anglais to babble, l'allemand babbeln, le coquillard babiller, avouer n'importe quoi... BL et BR en arrivent à se redoubler euxmêmes. Le mélange le plus hétéroclite ne s'exprime-t-il pas à travers les onomatopées en bloc et en blic $\left(15^{\mathrm{e}} \mathrm{s}\right.$.), à bricq et à bracq $\left(16^{\mathrm{e}} \mathrm{s}.\right)$, de bric et de broc $\left(17^{\mathrm{e}} \mathrm{s}\right.$.) et, bien entendu, le bric-à-brac enregistré par l'Académie en 1825 ?

\section{Séquences redoublées}

Quand l'onomatopée se répète deux, voire trois fois, c'est elle qui occupe tout le mot, le saturant de ses stigmatisations: un broubrou, en vieux lyonnais, est quelqu'un qui mélange tout, "qui dérange tout pour n'aboutir à rien » ${ }^{19}$; le Dictionnaire de l'Académie cite encore la vieille expression bredi-breda (sans articuler, à toute allure) : «Il nous a raconté cela bredi-breda. $»^{20}$ Tel est le cas moderne de blabla, voire blablabla, onomatopées pures des années quarante, "paroles creuses destinées à masquer le vide et à faire impression sur l'auditeur» (Robert historique). Origine: invention de Céline, affirme Albert Paraz. Mais Alice Becker-Ho nous apprend qu'on trouve blah-blah dans l'argot américain au sens de "propos creux, paroles pour ne rien dire " (à partir de blah, qui signifierait sornette, fadaise et, plus lointainement, du nom a blah qui désigne, en anglais populaire, « an indiscreet talker», baratineur assommant ${ }^{21}$ ). Avec la réduplication, le discours est signalé comme tellement inintéressant

17. J.-P. Dickes, 1976, On sommes péquailles, Boulogne-sur-Mer, Dickes, p. 90.

18. Belue, plus ancien $\left(13^{\mathrm{e}} \mathrm{s}\right.$.), serait un déverbal de beluer, éblouir, faire voir double (de bis lucere, hypothèse Diez).

19. N. de Puitspelu, 1903, Le Littré de la Grand-Côte, Lyon, À l'image de la cigogne, réédition Prolibra, 1988, p. 63.

20. Dictionnaire de l'Académie française, 1884, t.1, p. 218. À rapprocher de bret, cri mal articulé du corbeau dans le Roman de Renart (vers 929, 935) et sans doute de bredin (berdin en Bourbonnais), de bretonner, breter, bretete, brecton, voire bredindin, mauvais palan pour mauvais chargement: en instabilité permanente.

21. A. Becker-Ho, 1993, Les princes du jargon, Paris, Gallimard (Folio), p. 40. 
qu'on n'en constate que le bruit, qu'on le rejette comme étranger et qu'on pourrait supposer en son arrière une volonté d'entourloupe. Avec BRBR, en plus réaliste encore, on ne saurait oublier l'onomatopée grecque qui a donné, au $16^{\mathrm{e}} \mathrm{s}$., le français borborygme, gargouillis malséant. Avec BRBL ou BLBR, on peut brimbaler, brinquebaler ou bringuebaler, voire, avec Gulliver, bourlinguer dans la tempête jusqu'à Balnibarbi (BL BRB), ce pays où, comme en ordinateur, toutes les combinaisons de lettres sont possibles et où l'échange, renonçant à la parole, se pratique entre objets monosémiques, qui, eux au moins, ne mentent pas. On peut même trouver des réduplications inversées, telle BR LB. Témoins, aussi bien le maritime puis militaire branle-bas, agitation surexcitée, que le burluban, ce chêne magique des Celtes que hantent des voix de revenants:

Les uns et les autres se signalaient [...] par un étonnant et mystérieux concert. Aux cris rauques et stridents des oiseaux de mer attirés dans les parages se mêlait sur leurs branches le bruit provoqué par de petites «harpes rustiques». Ces instruments servaient alors de leurres pour attirer les animaux. [...] Le vent venait s'y engouffrer comme dans un sifflet d'enfant ${ }^{22}$.

Toutes ces évocations remuantes ou ces marques d'incompréhension ne seraient-elles pas présentes aux naissances multilingues de barbare?

Motif rédupliqué lui aussi, le sumérien bar-bar aurait déjà signifié étranger. L'akkadien barbaru désignait le loup... Un cri arabe chasse l'envahisseur: Bara! Bara! (Dehors! Dehors !) ; l'arabe barrani veut dire étranger. Une baraïta est, chez les rabbins, un enseignement resté «en dehors» de la Mishna23.

Côté indo-européen, le mot barbarah, en sanskrit, s'applique au bègue. Le grec barbaros réfère à tous les non-Grecs sans culture, indignes de la citoyenneté ${ }^{24}$; le barbarus latin (ou barbaricus), à ceux qui, n'étant ni Grecs ni Romains, sont vus comme étrangers, donc sauvages. Leur défaut le plus patent: ils parlent des langues incompréhensibles, celtiques, germaniques, parthes, perses, araméennes, tout juste bonnes pour le lamento des vaincus... Homère évoque «les Cariens à la voix barbare ${ }^{25}$. "Si tu n'as point compris mes paroles, réponds-moi par des gestes, comme les Barbares », dit Clytemnestre à Cassandre ${ }^{26}$, et le chœur des vieillards perses de supplier Darius en ces termes: «Le Roi égal aux Dieux m'entend-il pousser en langue barbare mille cris divers, amers, lamentables ? Je crie vers lui mes plaintes lugubres. $»^{27}$

Barbare est mentionné en français au début du $14^{\mathrm{e}} \mathrm{s}$., sans trop de péjoration. Comme en latin et en grec, il signifie surtout étranger, de langue bien

22. M. Duval, 2000, Mythologie des arbres en Bretagne, Mayenne, Royer, p. 66.

23. Féminin araméen signifiant extérieure (voir M. Haddas-Lebel, L'hébreu: 3000 ans d'histoire, Paris, Albin Michel, 1992, p. 53-54).

24. Exception, Ératosthène, refusant la distinction Grecs/Barbares: «Beaucoup de Grecs sont de méchantes gens et beaucoup de Barbares ont une civilisation raffinée».

25. Homère, L'lliade, traduit par E. Lasserre, 1955, Paris, Garnier frères, p. 43.

26. Leconte de Lisle, Eschyle, traduction nouvelle, Paris, A. Lemerre, s. d., Agamemnôn, p. 187.

27. Ibid., Les Perses, p. 344. 
entendu (Oresme: «Aucuns appellent barbares tous ceux qui sont de estrange langue »), mais aussi « étranger à la civilisation chrétienne d’Europe occidentale et à ses références antiques » (Robert historique), car les mots sont de leur temps. Au $16^{\mathrm{e}}$ s., si l'on en croit l'usage qu'en fait Nostradamus, le barbare étranger habite non plus au nord et à l'est, mais plutôt au sud, dans les pays du Maghreb, parfois dans le Moyen-Orient musulman ${ }^{28}$ : la classe (flotte) barbare, le sainct barbar (le fanatique), les barbarins, barbariques et autres barbaris ne cesseront, selon lui, d’infester dans l'avenir les ports méditerranéens :

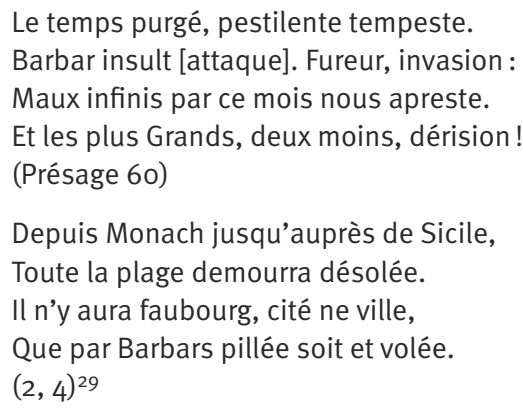

Barbare prend alors quatre voies différentes, qui se recoupent. La première, géographique, est marquée, on le voit, par la Barbarie, côte nord-africaine ${ }^{30}$ et pays arabes, mais aussi côte est de l'Afrique, dite en arabe côte d'Ajan ou, dans les traductions anciennes, Azania $^{31}$ (tandis que, pour les Romains, Barbaria nommait tous les peuples étrangers à la civilisation gréco-romaine, où qu'ils habitent).

Curieusement, c'est barbaresque (emprunté à l'italien barbaresco) qui, outre sa désignation des «États barbaresques» (qui allaient du Maroc à la Tripolitaine), a pu porter les valeurs «babéliennes» de langue de la confusion, donc de l'étranger. Edmond Huguet prend soin de nous prévenir, à propos d'un poème de Du Bartas ( $16^{\mathrm{e}} \mathrm{s}$.) : «Le sens que nous donnons aujourd'hui à barbaresque est venu beaucoup plus tard $»^{32}$.

28. Sauf, évidemment, lorsqu'il s'agit de l'histoire romaine. On connait le sonnet 30 des Antiquités de Du Bellay: «Ainsi, de peu à peu creut l'empire Romain,/Tant [jusqu'à ce] qu'il fut despouillé par la Barbare main.»

29. Voir J.-Ch. de Fontbrune, 1995, Nostradamus. Nouvelles prophéties jusqu'en 2025, Paris, Ramsay Marabout, p. 143-164.

30. La rapport entre Barbarie et Berbérie est-il de filiation ou d'analogie? On a pu rapprocher berbère (pluriel brabra) du barbaros grec (Homère infligeait aux Berbères l'épithète de barbarophares), mais aussi de l'arabe: ber, sol, friches, bariet, désert, barriyya, steppe ou savane, voire bara, côte, et bareia, terrestre (Joinville parle à leur propos des berries, landes à pâturages). Les Berbères, peuple de la terre (de bar, fils, bariyya, terre sauvage), par opposition aux «peuples de la mer» ? Voir F. Cheriguen, 1987, "Barbaros ou Amazigh. Ethnonymes et histoire politique en Afrique du Nord», Mots, $n^{0} 15$, octobre, p. 7-22.

31. La mer que longeait cette côte était nommée Azanium ou «mare barbaricum».

32. Avec les corsaires du $17^{\mathrm{e}} \mathrm{s}$. Voir Père Dan, 1637, Histoire de Barbarie et de ses corsaires. 
Muse, pardonne-moi si je pein de grotesques Un si riche tableau, si de mots barbaresques Je souille mon discours, veu qu'en cet argument Il faut, pour bien parler, parler barbarement ${ }^{33}$.

Dans cette acception, barbaresque et parler barbarement ont partie liée avec barbarisme, terme savant du $13^{\mathrm{e}} \mathrm{s}$., emprunté au grec d'Aristote avec la valeur d'" expression vicieuse », ou au latin barbarismus, "faute contre la langue ou la prononciation d'un mot latin» (Lebaigue). Le classicisme fera la chasse au « honteux barbarisme» (Boileau, Art poétique).

La troisième voie opère un revirement appréciatif. À l'encontre des oukases classiques (Boileau : «D'un seul nom quelquefois le son dur et bizarre/Rend un poème entier ou burlesque ou barbare »), c'est le temps de la préciosité, où barbare, à l'instar de cruel, devient un mot noble de la rhétorique amoureuse : «Donne-moi donc, barbare, un cœur comme le tien! »34. Viendront ensuite les renversements propres à l'époque romantique, «le barbare étant considéré comme vigoureux par rapport aux décadences des civilisations raffinées » (Le Robert). Silvia Disegni relève dans les articles Assassins des premiers Larousse (1863-1875) une suite d'épithètes analogiques qui commence par: barbare, cruel, farouche, horrible, hideux et se termine par: redoutable, célèbre, fameux, fier, héroïque ${ }^{35}$. Curieuse échelle, dont barbare parait être le barreau le plus bas. Mais ne pourrait-on pas dresser la même échelle pour barbare lui-même, qui trouverait là, dans l'admiration qu'il suscite, une revanche à ses stigmatisations ? À travers BR, barbare, burlesque, baroque, voire bravoure, se sont rapprochés ${ }^{36}$.

La quatrième voie, de loin la plus importante, conduit aux emplois infamants. On trouve, dans l'usage social de ces labels à la Renaissance, une présupposition bien installée d'anarchie et de sauvagerie. Citant le cas des habitants de la «France Antartique » et des «terres neuves» dans son chapitre sur «les cannibales», Montaigne s'élève contre la «voix commune», les «opinions vulgaires », bref les préjugés portés par les mots:

Il n'y a rien de barbare et de sauvage en cette nation, à ce qu'on m'en a rapporté, sinon que chacun appelle barbarie ce qui n'est pas de son usage. Comme de vray il semble que nous n'avons autre touche de la verité et de la raison que l'exemple et idée des opinions et usances du païs où nous sommes 37 .

33. E. Huguet, 1967, L'évolution du sens des mots depuis le XVIe siècle, Genève, Droz, p. 159.

34. Corneille, Horace, acte IV, scène 5.

35. Dans M. Piarotas, 2000, Regards populaires sur la violence, Saint-Étienne, Publications de l'Université, p. 49.

36. Notons la filière particulière de brave $\left(14^{\mathrm{e}} \mathrm{s}\right.$.), qui vient elle aussi du latin barbarus, par un vulgarisme supposé brabus et l'intermédiaire de l'italien bravo. Fier et courageux depuis le $16^{\mathrm{e}} \mathrm{s}$., le brave s'est spécialisé en bretteur au $17^{\mathrm{e}}$, puis est devenu simplement gentil, honnête homme (un brave homme, $18^{\mathrm{e}} \mathrm{s}$.). La bravoure ( $17^{\mathrm{e}} \mathrm{s}$., de l'italien bravura) est restée purement laudative. On l'applaudit à grand bruit, comme les Italiens : Bravo! (18 $\mathrm{s}$.).

37. 1938, Essais, Paris, Flammarion, livre 1, chap. 31, p. 245. 
Cette valeur dépréciative originelle envahit le discours au siècle suivant. Bossuet parle de la Thrace et de la Scythie comme de «peuples barbares et presque sauvages ». Évolution : non seulement barbare est devenu synonyme de cruel $^{38}$, mais il ne s'attache plus forcément à l'étranger; la barbarie s'illustre au sein d'une même famille, dans nombre de tragédies classiques: «Père barbare, achève, achève ton ouvrage!» (Corneille, Polyeucte, acte V, scène 5). Agrippine maudit Néron en prophétisant ses futurs crimes en série :

Tes remords te suivront comme autant de furies;

Tu croiras les calmer par d'autres barbaries;

Ta fureur, s'irritant soi-même dans son cours,

D'un sang toujours nouveau marquera tous tes jours.

(Racine, Britannicus, acte V, scène 6)

Et les «invasions barbares", les «royaumes barbares», avec leurs "vandalismes », de submerger l'Europe jusque dans les manuels d'histoire ${ }^{39}$; Chateaubriand les évoque des hauteurs de Dunkeim :

Burgondes, Francs, Goths, Huns, Suèves, flots du déluge des Barbares, ont tour à tour assailli ces hauteurs. [...] Les Barbares nos pères égorgèrent, à Metz, les Romains surpris au milieu des débauches d'une fête ${ }^{40}$.

C'est dans cette acception d'envahisseurs sauvages mais reconnus puissants, redoutables et courageux, que le mot déplace le plus son champ d'application. À la suite de la révolte des canuts lyonnais de novembre 1831, Chateaubriand risque, entre eux et les anciens «barbares » capables «d'imposer des tributs aux empereurs», une comparaison que le Journal des débats développe en décembre 1831 sous la plume de Saint-Marc Girardin :

La sédition de Lyon a révélé un grave secret, celui de la lutte intestine qui a lieu dans la société entre la classe qui possède et celle qui ne possède pas. Notre société commerciale et industrielle a sa plaie comme toutes les autres sociétés; cette plaie, ce sont les ouvriers. [...] Les barbares qui menacent la société ne sont point au Caucase ni dans les steppes de la Tartarie; ils sont dans les faubourgs de nos villes manufacturières. [...] Ils souffrent ; la misère les écrase. Comment ne seraient-ils pas tentés d'envahir la bourgeoisie? Ils sont les plus forts, les plus nombreux. [...] Il faut que la classe moyenne sache bien quel est l'état des choses. Elle a au-dessous d'elle une population de prolétaires [...] C'est là où est le danger de la société moderne; c'est de là que peuvent sortir les barbares qui la détruiront ${ }^{41}$. [Je souligne]

38. Le prophète, chez Garnier, apostrophe ainsi Nabuchodonosor: " 0 barbare cruel, homme avide de sang,/Qu'une tygre félonne a porté dans son flanc [...]/Que tu es impiteux, que tu es sans merci! » (Les Juives, acte V, p. 91)

39. «Grandes invasions » : «nom donné aux migrations de peuples barbares, en majorité des Germains qui, fuyant devant les Huns (375 apr. J.-C.) pénétrèrent dans l’Empire romain » (D. Vallaud, 1975, Dictionnaire historique, Paris, Fayard, p. 481-482).

40. 1833, dans les Mémoires d'outre-tombe, 1964, Paris, Gallimard (La Pléiade), t. 2, p. 742, 747.

41. Cité dans F. Rude, 1967, C'est nous les canuts, Paris, Maspero, p. 238-239 et M. Moissonnier, 1988, Les canuts, Paris, Messidor, p. 138. 
Barbare ressort de cette histoire profondément marqué par la violence, dans une opposition frontale à la bonne civilisation (ou au confort bourgeois). On a aujourd'hui le choix: "socialisme ou barbarie», "démocrates ou barbares". Certes, c'est la «barbarie nazie » qui a rendu le mot totalement odieux ${ }^{42}$. Mais ne l'était-il pas dès l'origine?

Avec ces séquences bilitères, trilitères ou quadrilitères du type $B R, B L, B R B$, $B L B, B R B R, B L B L$ et leurs innombrables variantes, sommes-nous en présence de racines linguistiques? Deux observations, en prenant le cas de $B L$ :

- ces dites "racines» se retrouvent bien au-delà de l'indo-européen, ne serait-ce que dans Babel et Babylone (BBL), lieux de l'unité rêvée ou de l'uniformité imposée, impermanentes toutes deux, puis de la confusion, de la diversification des langues et de l'incompréhension entre peuples dispersés (Genèse, 11), noms sémitiques s’il en est;

- elles se superposent (ou se sub-posent) à d'autres formants, eux proprement linguistiques : Babel, Babylone lient bab (la porte) à Ilu (en akkadien) ou El (en hébreu) : Dieu, et à llâni (en hébreu) : les dieux. Il y a même, dès l'origine, une troisième sémantisation: le verbe hébreu bâlal (BLL), interprété comme mêler, confondre : «Elle [la tour] reçut le nom de Babel, car c'est là que le Seigneur mit la confusion dans le langage de tous les habitants de la terre» (Genèse, 11, 9). Barbare, indo-européen, et Babel, sémitique, auraient source commune, à l'origine de dires mal prononcés et mal ouïs tel le babil enfantin...

Structurantes en arrière-plan, à l'évidence, ces pseudo- «racines», mais non clairement signifiantes: elles manifestent des émotions intellectuelles (incompréhension), des appréciations péjoratives (sottise, inculture, tromperie, violence, cruauté), des sensations auditives (confusion des bruits et des sons), visuelles (berlue, bariolage), tactiles (ébranler, brutaliser), voire gustatives ${ }^{43}$, des thèmes séparatistes (la «divergence» selon P. Guiraud, l'exclusion). Leur seul aboutissement à peu près désignatif: l'étranger à l'homme, au citoyen, à nous, à soi, vu comme adversaire de nature ${ }^{44}$. Mieux qu'une racine «mondiale» (c'est-à-dire conforme aux hypothèses de Merritt Ruhlen) ${ }^{45}$, ne s'agirait-il pas, tout au fond des paroles émises, de la manifestation d'un substrat phonosémique porteur d'une infinité d'engendrements possibles et qui accompagnerait d'une motivation inconsciente l'arbitraire des

42. Et cela d'autant plus qu'elle revendiquait, par retorsion, le label: «Nous sommes les nouveaux barbares, nous sommes la jeunesse du monde », criait Hitler (cité dans J.-C. Guillebaud, 2003, "La barbarie invisible», La Vie, $\mathrm{n}^{\circ}$ 3002, 13 mars, p. 37).

43. Tels les grands mélanges d'herbes et de gousses provençales dans les mets «à la barigoule».

44. Platon distinguait guerre et discorde, la guerre se faisant entre Grecs et Barbares (car « ils sont ennemis par nature ») et la discorde entre Grecs (car «ils sont amis par nature »). Voir Platon, 1966, La République, traduit par R. Baccou, Paris, Garnier-Flammarion, p. 226.

45. M. Ruhlen, 1997, L'origine des langues. Sur les traces de la langue mère, Paris, Belin. 
signes et l'histoire des étymons ? $^{46}$ Telle une musique qui resterait potentiellement créative. Les archaïques sobriquets latins babulus et baburrus (babillard ou niais), si proches de barbaros 47 comme de babélien, ne se réactivent-ils pas dans les modernes babil et blablabla? Les appréciations à contre-courant, laudatives, en demeurent fragiles, tant le substrat péjoratif est tenace: au départ éloge du mélange sans racisme et de la confusion bénéfique, méfionsnous de certains usages larvés de black-blanc-beur (BL BL BR).

Proches parents des protosémantismes et des «motivations onomatopéiques » proposés par P. Guiraud, n'existerait-il pas des protophonologismes plus fondamentaux que les étymons? BR/BL ou le «signal» de la parole incomprise et de la division des hommes. Il faut la lucidité de Montaigne pour ne pas se laisser prendre à leurs jeux inconscients.

46. Bonne occasion pour relire Michel Foucault... et Court de Gébelin (voir M. Foucault, 1966, Les mots et les choses, Paris, Gallimard, NRF, p. 118-125).

47. A. Meillet et J. Vendryes rangent ces «noms à redoublement» parmi les «noms exprimant le bruit, le bouillonnement, le fourmillement, la masse» (1960, Traité de grammaire comparée des langues classiques, Paris, Champion, p. 370). 